\title{
Tuberculosis mastitis - Still a Great Masquerader
}

\author{
Dr Rahul R Bhat ${ }^{1}$, Dr Shibumon M M², Dr Prathibha Naik ${ }^{3}$,Dr Shankar Ram ${ }^{4}$ \\ Dr Shivananda Prabhu ${ }^{5}$, Dr Manohar Pai ${ }^{6}$, Dr Poornachandra Thejeswi ${ }^{7}$ \\ ${ }^{1}$ (Assistant Professor, Department of General Surgery, KMC Mangalore, Manipal University, India) \\ 2 (Assistant Professor, Department of General Surgery, KMC Mangalore, Manipal University, India) \\ ${ }^{3}$ (Resident, Department of General Surgery, KMC Mangalore, Manipal University, India) \\ ${ }^{4}$ (Senior Resident, Department of General Surgery, KMC Mangalore, Manipal University, India) \\ ${ }^{5}$ (Professor, Department of General Surgery, KMC Mangalore, Manipal University, India) \\ ${ }^{6}$ (Additional Professor, Department of General Surgery, KMC Mangalore, Manipal University, India) \\ (Associate Professor, Department of General Surgery, KMC Mangalore, Manipal University, India)
}

\begin{abstract}
Though India has major burden of cases of tuberculosis in the world, tuberculous mastitis remains to be one of the least studied entities. In this retrospective study we have emphasised clinical and demographic profile of patients diagnosed with tuberculous mastitis and their response to anti tubercular therapy. We had 10 patients diagnosed with tuberculous mastitis during the period of 2009 - 2014. All patients were in reproductive bracket commonly presenting in the age group of 30 - 35 yrs. Presentation involved equal frequency in both breasts and duration spanned over 2 days to a year. Most of them were house wives and parous women who had breast fed children. Usual presentation was breast lump whose mean size was $4.5 \mathrm{cms}$ associated with dull pain. Diagnosis was confirmed by histopathology which showed granulomatous inflammation, caseating necrosis with or without demonstrable tuberculous bacillus. Patients were treated with anti tubercular therapy for 6 months resulting in complete cure of disease with no relapse or recurrence.
\end{abstract}

Key words: Anti tubercular therapy, Granulomatous mastitis, Tuberculous mastitis, Tuberculosis(TB),

\section{Introduction}

Chronic granulomatous mastitis is more prevalent in developing countries and has been predominantly tubercular in origin. Historically Sir Astley Cooper is credited to have described the condition as early as in 1829 which he described as scrofulous swelling at the bosom of young women. Prevalence of tuberculosis (TB) in Indian population is 3.1 million. It is estimated that about $40 \%$ of the Indian population is infected with TB bacteria, the vast majority of whom have latent rather than active TB. With one of the highest TB burden in the world we expect a large subset of populace to be suffering from tuberculous mastitis. ${ }^{3,4}$. Incidence of tuberculous mastitis in India has been between $1-4.5 \%$.In our study we have tried to emphasise importance of subtle clinical signs and symptoms and demographic profile that distinguishes them from carcinoma of breast. Also we have come to understand the relevance of Anti tubercular therapy (ATT) in the absence of demonstrable bacillus. It has been observed that the disease is common in reproductive age group in women due to increased vascularity following lactational status ${ }^{4,5}$.

The most common symptom has been observed to be lump in breast. Breast lump remains mobile until it arises secondarily from chest wall ${ }^{15}$. Also a patient with discharging sinuses, ulcer and matted axillary lymph nodes raises the probability of suspicion ${ }^{5,6,7}$. Less than $50 \%$ have all the above mentioned constellation of signs and symptoms. There is no preponderance related to side and bilaterality is rare ${ }^{15}$. Symptoms and signs have known to occur of varied duration usually of few weeks to several months and occasionally in to years.

Amongst the various risk factors considered to be associated with tuberculous mastitis are multiparity ${ }^{12}$, lactation $^{13}$, trauma ${ }^{13,14}$ and past history of suppurative mastitis.

It has been hypothesized that involvement of breast is often secondary to undiagnosed infection elsewhere in the body. The common portal of spread has been ascribed to retrograde spread from axillary or internal mammary lymph nodes ${ }^{16}$. Primary tuberculosis of breast is a rarity and often a result of traumatic inoculation.

\section{Materials And Methods}

Study incorporates retrospective analysis of patients who had histological diagnosis of granulomatous disease with or without tuberculosis over 5 years. Initial assessment of any patient with complaints related to breast includes the standard protocol of triple assessment i.e. clinical examination, imaging and cytology. The diagnosis was often a histological surprise. Histological diagnosis was achieved either in form of Fine needle 
aspiration cytology (FNAC) conducted for breast swellings or biopsy for patients who presented with breast abscess. In some cases with nipple discharge we have considered imprint smears.

Pathology reporting was done in Department of Pathology, Kasturba Medical College, Mangalore. In case of abscesses Zeil - Neilson (ZN) staining for acid fast bacillus (AFB) was considered. In one case Real time polymerase chain reaction (PCR) for TB antigen was done. ${ }^{7}$ Diagnosis was by demonstration of bacillus in the aspirate or histopathology showing presence of granulomatous inflammation with caseating tubercles. All patients underwent sonomammogram. Chest $\mathrm{x}$ ray and sputum for AFB were carried out to check for primary pulmonary TB once the diagnosis of tuberculous mastitis was made.

\section{Results}

In our study 54 patients who had inflammatory disorders of breast were considered. Among them 10 were cases diagnosed with granulomatous mastitis.

TABLE - 1.Age distribution of granulomatous mastitis (n - 10)

\begin{tabular}{|l|l|l|l|l|l|l|l|}
\hline $\begin{array}{l}\text { Age } \\
\text { group }\end{array}$ & $15-20 \mathrm{yrs}$ & $21-25 \mathrm{yrs}$ & $26-30 \mathrm{yrs}$ & $31-35 \mathrm{yrs}$ & $36-40 \mathrm{yrs}$ & $41-45 \mathrm{yrs}$ & $46-50 \mathrm{yrs}$ \\
\hline Cases & 0 & 0 & 2 & 4 & 3 & 0 & 2 \\
\hline
\end{tabular}

Six amongst them were house wives and one was a maid. Occupational history of other 3 is unknown. There were no cases of Diabetes Mellitus, HIV and none had history of trauma.

TABLE-2. Duration of the condition

\begin{tabular}{|c|c|c|c|}
\hline$<1$ week & 1 week -1 month & 1 month -2 month & $>2$ months $-<1$ year \\
\hline 3 & 1 & 2 & 4 \\
\hline
\end{tabular}

TABLE-3 Disease pattern signs and symptoms

\begin{tabular}{|c|c|c|c|c|c|c|c|}
\hline \multirow{3}{*}{$\begin{array}{c}\text { Total number } \\
\text { of cases }\end{array}$} & \multirow{2}{*}{\multicolumn{2}{|c|}{$\begin{array}{c}\text { swelling } \\
11 \\
\end{array}$}} & \multirow{3}{*}{ pain } & \multirow{3}{*}{ nipple discharge } & \multirow{3}{*}{ sinus } & \multirow{3}{*}{ abscess } & \multirow{3}{*}{$\begin{array}{c}\text { axillary lymph } \\
\text { nodes }\end{array}$} \\
\hline & & & & & & & \\
\hline & Right & Left & & & & & \\
\hline 10 & 5 & 5 & 10 & 1 & 2 & 7 & 1 \\
\hline
\end{tabular}

Size

Size of the swellings varied from $2-10 \mathrm{~cm}$. Average size of the swelling was $4.5 \mathrm{cms}$.

TABLE 4 -Total number of cases of granulomatous mastitis $=10$

\begin{tabular}{|c|c|}
\hline TB+ve & TB-ve \\
\hline 3 & 7 \\
\hline
\end{tabular}

TB bacillus was demonstrated by FNAC alone in one case. Out of three patients one required real time PCR for TB antigen. Pus sent for Zeil-Neilson staining helped in detection in a young female of 27 yrs, whereas histopathology picked up the bacillus in another female aged $47 \mathrm{yrs}$. All patients had undergone chest $x$ ray and sputum examination. None of them were deemed to harbor underlying pulmonary tuberculosis.

\section{Treatment and follow up}

Antitubercular therapy was started in 3 patients who had demonstrable bacteria whereas it was also started in other 7 patients who had histopathological diagnosis of granulomatous mastitis showing caseating tubercles. ${ }^{7}$ All patients were found symptom free apart from cicatrised lesion at the end of therapy. As yet there has been no report of recurrence in any form from the mentioned group of patients. Maximum follow up period was 5 years. 


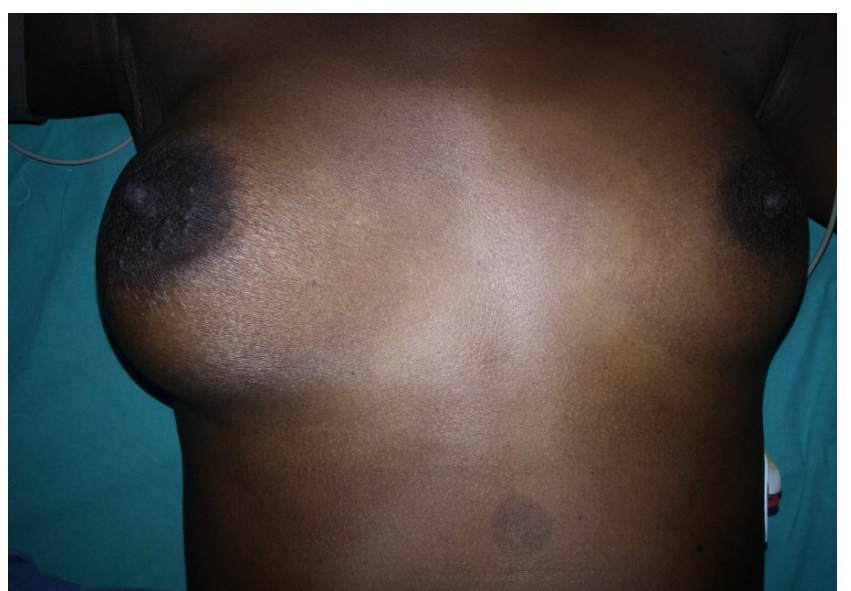

Figure 1: Secondarily infected tubercular abscess of right breast

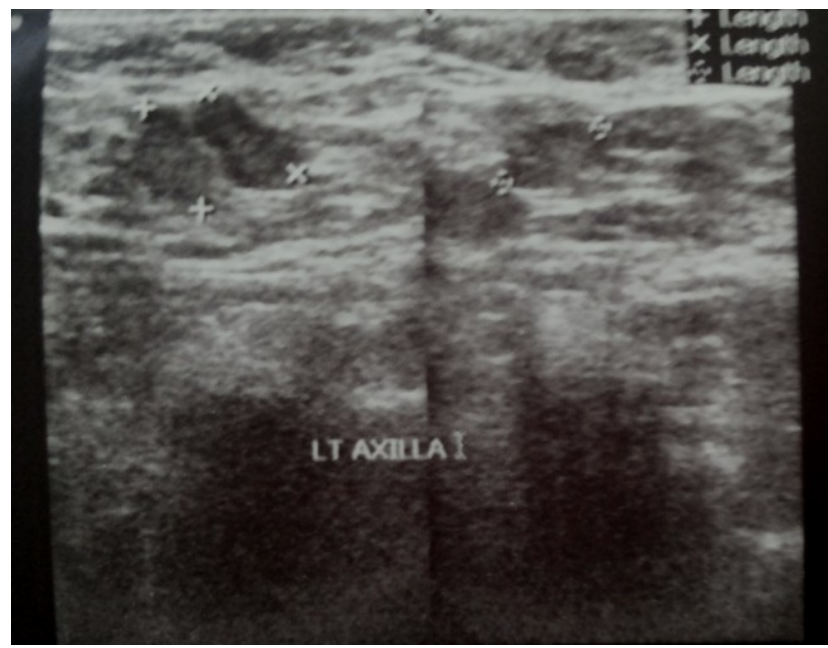

Figure 2: Sonomammogram of a $32 \mathrm{yr}$ old patient who presented $6 \times 8 \mathrm{~cm}$ mass showing multiple small collections within the mass. FNAC reported granulomatous mastitis and real time PCR was positive for TB antigen.

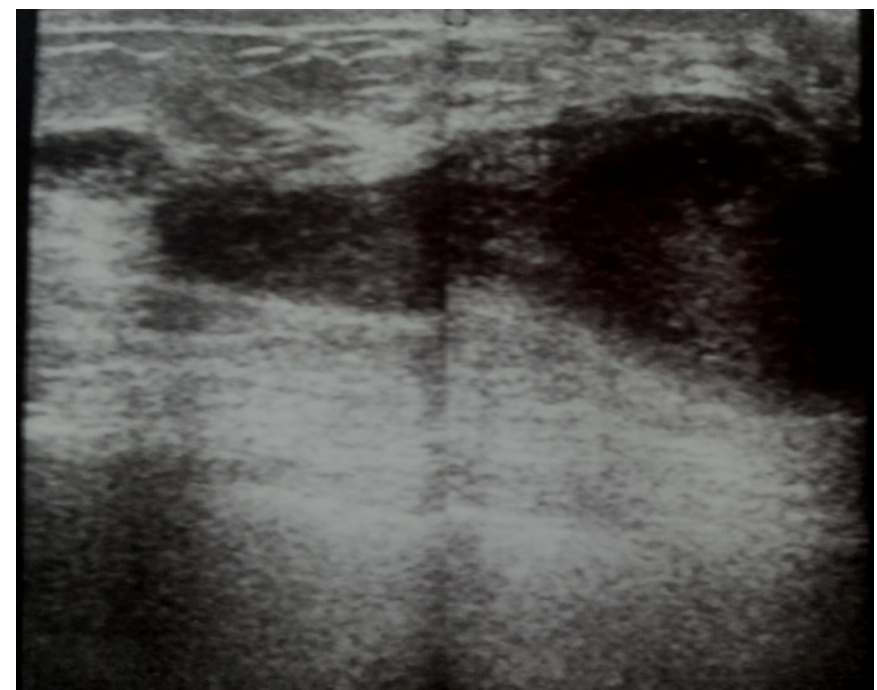

Figure 3: The same patient after three months reported with increasing pain. Sonomammogram showed a large collection suggesting cold abscess. Decision was taken to manage conservatively and to continue with anti tubercular therapy. Patient is currently symptom free. 


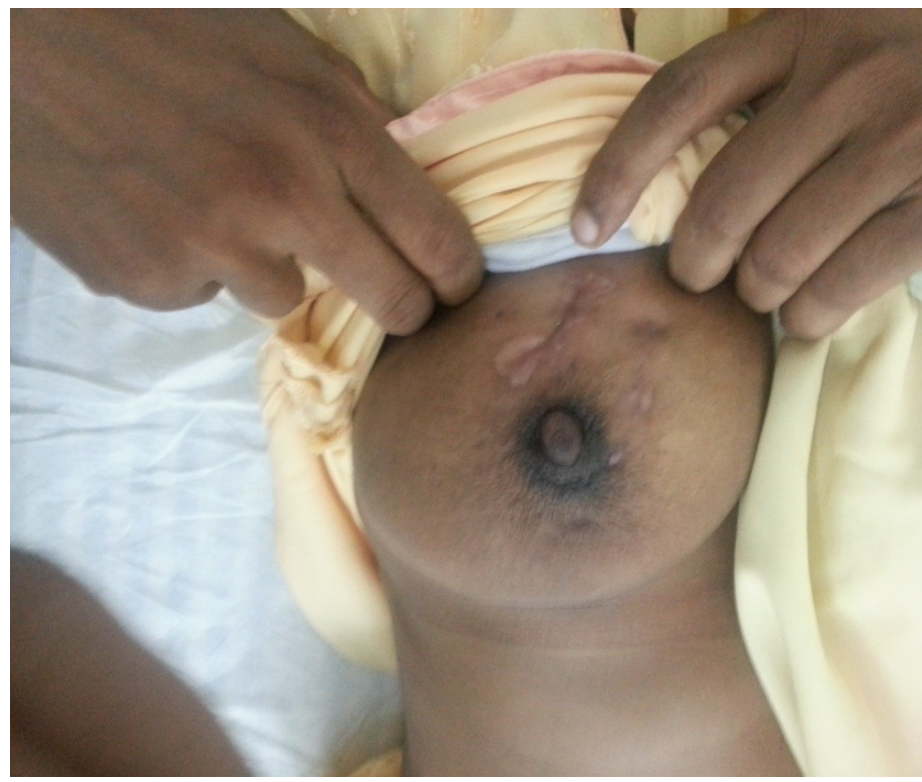

Figure 4: Healed tubercular abscess

\section{Discussion}

In India incidence of tuberculous mastitis is in range of $1-4.5 \%$ of mammary lesions $\mathrm{s}^{3,4,5}$. Overall world wide incidence is $0.6-1.6 \%$. In our study we found granulomatous mastitis attributing to $18.5 \%$ of inflammatory conditions seen in our hospital. Inflammatory conditions included pyogenic abscess of breast, giant cell and plasma cell mastitis. Considerably higher incidence was attributed to it's presentation as a mass only, mimicking malignancy and hence formally referred to our setup for further management. Granulomatous mastitis mimicking malignancy is a well known entity and in popular medical literature it has been called as "a Great Masquerader" especially in older individuals. ${ }^{1,2}$

Most common symptom according to literature is swelling, the size and nature resembling carcinoma breast. Though these swellings are mobile they tend to exhibit fixity to overlying skin. Their mobility is no match to that of a fibroadenoma as invariably they involve ligaments of Cooper displaying characteristic restricted mobility mimicking malignancy. Sinuses are commonly found in long standing untreated cases.

In our study we had 2 patients with discharging sinuses. Involvement of axillary lymphnodes as lymphadenopathy with or without matting can be expected.

Dull aching pain in the involved breast distinguishes the condition from malignancy. Presence of pain was a consistent factor in our study. All 10 patients had dragging type of pain which increased upon stooping and activity which relieved on wearing tight brassiere. Incidence of presentation in both breasts was equal. ${ }^{4,11}$ Several studies support the same.

In our study we have found that the condition tends to predominantly affect females in reproductive age especially in women aged between $30-40 \mathrm{yrs}$. Nine of them were married and parous. ${ }^{11}$ In the study conducted by Nadir Mehmood et al the mean age of presentation was $32.8 \mathrm{yrs}$ and $53 \%$ were parous. Hence it is more common in women who have borne and breast fed children. ${ }^{6}$ It has also been noticed that tuberculosis of breast has been found to be relatively uncommon in older women and prepubertal girls. We had no patient out of reproductive bracket.

None of the cases had contact history. Curiously neither of the patients had history of primary tuberculosis either in lungs or elsewhere. Chest $\mathrm{X}$ ray and sputum examinations were unremarkable also patients did not have cervical lymphnodes. Reviewing the cases led us to believe that atleast some of them were primary, the only way to prove will be a prospective study. ${ }^{8}$ Mckeown and Wilkinson had classified breast tuberculosis as primary when it was the only manifestation and secondary when a demonstrable focus of tuberculosis was found elsewhere in the body. ${ }^{9}$ Vassilakoss opined that breast being resistant tissue to tubercular infection, primary tuberculosis of breast was rare and was labelled as primary as clinician was unable to detect the true focus of disease. Lymphadenitis in axilla was seen in only 1 case. ${ }^{6,7}$ Usual scenario suggests presence of axillary lymphnodes in one third of cases.

Earlier, surgery used to be the mainstay of treatment where infected tissue was resected. Nowadays anti tubercular therapy with limited surgical intervention or aspiration of abscess has been found to be sufficient ${ }^{13}$. In our study one patient required repeated aspiration; initially at diagnosis and repeated after 3 months. Sector mastectomy was conducted in a lady of 26 yrs age due to intractable pain. Both patients responded well to anti tubercular therapy. 
Anti tubercular therapy included an intensive phase of 2 months with 4 drugs followed 4 months of continuation phase with 2 drugs [2HRZE/4HR]. This is in accordance with category 1 regimen of DOTS-ATT programme in India. The therapy included not only patients who tested positive for TB bacillus but also patients with granulomatous mastitis with caseating tubercles but no demonstrable bacteria. All 10 patients were symptom free by the end of therapy of 6 months and reported no relapse which was ascertained by timely follow up and telephonic interview.

\section{Conclusion}

Any patient in reproductive age group who is non lactating and has presented with tender breast mass of short duration, it will be a justified decision to investigate for tuberculosis especially in presence of a discharging sinus. Anti tubercular therapy for granulomatous mastitis with caseating necrosis in absence of conclusive evidence of bacillus is justified in our study.

\section{References}

[1]. Popli M.B. Tuberculosis of the breast. Ind J Radiological Image.1999;9:127-132.

[2]. Zahra F, Jafferys. Tuberculosis mastitis. Mother child.1998; 36: 146- 148

[3]. Khanna R, Prasanna GV, Gupta P, Kumar M, Khanna S, Khanna AK. Mammary tuberculosis: report on 52 cases. Postgrad Med J 2002. Jul;78(921):422-424.

[4]. Aggarwal V, Bhargava P. Breast tuberculosis-a case report. J Indian Med Assoc 2008. Jan;106(1):38-40, 40.

[5]. Puneet, S Tiwary, R Ragini, S Singh, S Gupta, V Shukla. Breast Tuberculosis: Still Common In India. The Internet Journal of Tropical Medicine. 2004 Volume 2 Number 2.

[6]. Shinde SR, Chanda Warker RY, Deshmukh S.P. Tuberculosis in breast masquerading as carcinoma. A study of 100 patients . World J.Surgery 1995; 19:379-381

[7]. Jalali, U, et al .Tuberculous mastitis -J.C.P.S.P. 2005 vol.15(4) :234- 237

[8]. Mckeown KC, Wilkinson KW. Tuberculous diseases of the breast. Br J Surg 1952; $39: 420$

[9]. Vassilakos P. Tuberculosis of the breast: cytological findings with fine-needle aspiration. A case clinically and radiologically minicking carcinoma. Acta Cytol 1973; $17: 160-5$.

[10]. Cooper. A. Illustrations of Diseases of the breast: Part I. London,England: Longman, Orme, Brown and Green ;1829:73

[11]. Mehmood N, Zeeshan HK, Umer AK, Aamir N, Malik IA, Khan MI. Tuberculous mastitis-presentation and outcome in our set up. Ann Pak Inst Med Sci 2009; 5(4): 245-250.

[12]. Anspach BM. Primary tuberculosis of the breast. Am J Med Sci 1904; 128: 98

[13]. Webster CS. Tuberculosis of the breast. Am J Surg 1939; 45: 557

[14]. Gilbert AL McGough EC. Farrell JJ. Tuberculosis of the breast. Am J Surg 1962; 103: 424

[15]. Banerjee SN, Ananthakrishnan N, Mehta RB, Parkash S. Tuberculous mastitis: a continuing problem. World J Surg 1987. Feb;11(1):105-109.

[16]. Schaeier G. Tuberculosis of the breast, a review with the additional presentation often cases. Am Rev Tuberc \& Pulm Dis 1955; 72 : 810 . 